\title{
Traffic Flow Modeling of Freeway Variable Speed Limit Control Based on the Big Data of Driving Behavior
}

\author{
Xu Qu $\mathbb{D}^{1,2}$ Linheng Li, ${ }^{1,2}$ Ziwei Yi, ${ }^{1,2}$ Peipei Mao, ${ }^{1,2}$ and Mofeng Yang $\mathbb{D}^{3}$ \\ ${ }^{1}$ School of Transportation, Southeast University, Nanjing, China \\ ${ }^{2}$ Jiangsu Province Collaborative Innovation Center of Modern Urban Traffic Technologies, Southeast University, Road \#2, \\ Nanjing 211189, China \\ ${ }^{3}$ Department of Civil and Environmental Engineering, University of Maryland, College Park, MD 20742, USA
}

Correspondence should be addressed to Xu Qu; quxu@seu.edu.cn

Received 22 March 2020; Revised 22 June 2020; Accepted 30 June 2020; Published 20 July 2020

Academic Editor: Weiwei Qi

Copyright (c) $2020 \mathrm{Xu}$ Qu et al. This is an open access article distributed under the Creative Commons Attribution License, which permits unrestricted use, distribution, and reproduction in any medium, provided the original work is properly cited.

\begin{abstract}
Variable speed limit (VSL) control is a flexible restriction on the rate at which motorists can drive on a given stretch of road. Effective VSL control can increase safety and provide clear guidance for motorists. Previous traffic flow models of VSL control were mostly based on the influence of VSL on average speed (macro) or driver's expected speed (micro). Few models considered the influence of VSL on driver's actual driving behavior. In this paper, we first briefly introduce the big traffic data involved in this study and explain the mapping relationship between the data and driving behavior. Then, we analyze the driver's actual driving behavior under the VSL control. Then, an improved single-lane cellular automaton model is established based on the driving behavior characteristics under VSL control. After that, we calibrate the parameters of the single-lane cellular automaton model with the left lane as the calibration object. Finally, this paper uses the proposed single-lane cellular automaton model to simulate the traffic flow characteristics under VSL control. The numerical simulation results show that the simulation of the variable speed limit in different density intervals presents different results, but these results are consistent with the actual situation of variable speed limit control, which verifies the validity of the proposed model.
\end{abstract}

\section{Introduction}

The variable speed limit (VSL) control system adjusts the road speed limit in real time based on the traffic flow state detected by advanced traffic flow and environment detection technology. As one of the advanced highway management control methods, VSL has been applied in Western countries such as Germany, the United States, Netherlands, the United Kingdom, Finland, Israel, New Zealand, and Australia [1]. The implementation effect indicates that VSL has a great impact on improving road traffic safety and alleviating traffic congestion [2-5].

The essential basic research content of VSL is traffic flow modeling. Macroscopic models are an important component of VSL modeling, which can be divided into two categories in terms of the characteristics of different VSL traffic flows [6-9]. One category is well studied, considering the influence of VSL on the equilibrium speed relationship [10-14]. The equilibrium speed function is replaced by a new one with the VSL control parameter according to the modified fundamental diagram under VSL control. Another category establishes the macroscopic model using the correspondence between macroscopic and microscopic parameters when simulating the driver behavior under VSL control.

The macroscopic model has the advantage of high operational efficiency in numerical simulation, but most of the existing macroscopic models lack the behavior description of individual vehicles, which makes it impossible to simulate the safety benefits of VSL. Hence, the optimization goal of VSL is limited to efficiency.

The microscopic model is preferred because both the efficiency benefits and security benefits of VSL strategies can be evaluated. Lee et al. [15] used PARAMICS to simulate 
VSL control; Torday and Bierlaire [16] modeled the VSL microscopic model based on the MITSIM simulator; and Park and Yadlapati [17] used VISSIM to model the VSL with different compliance rates. Other microscopic traffic simulations of Cruise Control (CC) and Adaptive Control (ACC) are used [18-20]; such models were used for freeway traffic for the evaluation of the effectiveness of VSL [21, 22]. However, in most of the microscopic models, the phenomenon that VSL affects the vehicle's desired speed is pondered, but the changes in drivers' behavior are ignored. In fact, VSL performance depended greatly on compliance rates [23]; the VSL not only changes the desired speed but also changes the following behavior of drivers. This is reflected in a previous paper where the proportion of the smaller headway was reduced [24]. Besides, models on considering the desired speed are not reasonable. In most of the studies, vehicles' speed was set equal to the VSL value, which does not correspond with the real estimation. When simulating large or small value of VSL control, the desired speed would be slightly above or beneath the limit value, which is worth exploring but had not been investigated in existing models. Therefore, it is necessary to explore the impact regularity of VSL control under different traffic conditions and compliance rates.

Traffic data always plays an important role in the process of traffic flow modeling; especially in the research of microscopic traffic flow modeling, it is necessary to describe driving behavior characteristics through massive microscopic traffic data. Recently, many scholars have tried to use driving behavior data as a research entry point to solve a series of related transportation problems. Li et al. [25] analyzed drivers' deceleration behavior based on naturalistic driving data and, finally, a brake control system was designed. In order to evaluate whether a driving behavior is fuel-efficient, Hao et al. [26] mined the traffic operating data and fuel consumption data and finally built a corresponding analysis model. Deng et al. [27] established a curve safe speed model on the basis of a thorough analysis of driving behavior data.

The use of empirical data to investigate driving behaviors with VSL control was well-studied [10, 12, 17]. The consensus emerging from these studies is that VSL systems can improve safety by decreasing the mean speed value, the speed difference, and the percentage of small-time headway and can increase the mean time headway. Studies using empirical traffic data as input can objectively analyze the impacts of VSL on driving behaviors and derive credible conclusions. However, with the constraint of predefined VSL control strategies, the majority of these studies only have access to data from a limited range of traffic conditions and limited VSL values. Therefore, most of the researchers merely performed qualitative comparisons between driving behavior parameters whether they used VSL control or not while missing the quantitative examination of the relationship between traffic variables and VSL values. In a realworld implementation, VSL values vary in accordance with the prevailing traffic conditions and weather conditions. However, few scholars evaluated the potential outcomes of each VSL value. In the meantime, only a limited number of dissertations controlled traffic states when examining VSL impacts; therefore, it is difficult to determine whether the obtained results are caused by VSL control or by the difference in traffic states within the collected empirical data.

This paper summarized the literature on traffic flow models and driving behavior under VSL control and proposed an improved cellular automaton model that represented individual driving behavior and reproduced corresponding traffic flow characteristics under VSL control. The rest of this paper is organized as follows. Driving behavior under VSL control is analyzed with data from a motorway in Section 2. Section 3 establishes the single cellular automaton model taking account of the compliance parameter. In Section 4, empirical data of a highway segment is used to identify the distribution of driver's individual radical degree and calibrate the parameters of the proposed model. In Section 5, numerical simulation is conducted to validate the superiority of the proposed model in reproducing traffic flow characteristics under VSL control.

\section{Big Data Analysis of Driving Behavior}

2.1. Data Preparation. The data used in this study was collected from a two-way European motorway segment with three lanes in each direction. The traffic flow state changes obviously, including recurrent and nonrecurrent congestions. The traffic flow information, such as the vehicular speed, the headway, and the vehicle length, was collected by loop detectors installed every 500-600 $\mathrm{m}$ in each lane. In order to alleviate congestion and improve driving safety, the expressway was equipped with VSL control facilities that adopt a control strategy based on mitigating traffic shocks. A wide variety of speed limits including $50 \mathrm{~km} / \mathrm{h}, 60 \mathrm{~km} / \mathrm{h}$, $80 \mathrm{~km} / \mathrm{h}, 100 \mathrm{~km} / \mathrm{h}$, and $120 \mathrm{~km} / \mathrm{h}$ were implemented in the system.

This research collected traffic flow data and corresponding VSL control speed data from 7:00 AM to 6:00 PM for two weeks at a nonweaving area site. Only data in good weather condition (no rain or fog) and visibility conditions (at daytime) was selected to avoid external interference. Missing and wrong data was removed. Finally, the dataset including 4,266 minutes of data samples with 355,599 vehicles was constituted.

The traffic state is a crucial factor when investigating the VSL impacts on driving behavior. Under various traffic states, driving behavior will be differently affected by the VSL system. Hence, it is difficult to determine whether the obtained results are caused by the VSL control or by the difference in traffic states through the raw empirical data. To eliminate the impact of traffic states, the classification of the samples according to traffic states is indeed critical. In this paper, the collected samples were firstly classified into different traffic density intervals to measure traffic congestion. Then the traffic data in the same density interval with different VSL control was compared to that without VSL control to investigate the effect of VSL control on driving behavior. 
2.2. Desired Speed Distribution. From the previous study, it can be inferred that the VSL has the ability to reduce the desired speed, since the average speed in each density interval under free-flow state was descended with different VSL values. Gartner's study mentioned that when the space headway is greater than $125 \mathrm{~m}$ (corresponding to the headway of $4 \sim 6 \mathrm{~s}$ at $20 \sim 30 \mathrm{~m} / \mathrm{s}$ vehicle speed), the vehicle will not be in the following state [28]. According to the conclusion, vehicle speed in the above-mentioned state reflects the driver's desired speed, which is not affected by the preceding vehicle. A statistical analysis of the vehicle speed with a headway greater than $5 \mathrm{~s}$ in the free-flow state was performed. Figure 1 shows the vehicle speed distribution of the left lane in the uncontrolled state and VSL control state including $120 \mathrm{~km} / \mathrm{h}, 100 \mathrm{~km} / \mathrm{h}$, and $80 \mathrm{~km} / \mathrm{h}$.

Figure 1 demonstrates that the effect of the VSL on the driver's desired speed is holistic. After setting VSL, the driver basically responded and reduced the speed. The exponential regression line in the figure shows that the driving speed gradually increases as the headway increases, which is in line with reality. At the same time, it can be seen from the figure that under different VSL values, a few drivers drive at a speed obviously higher than the VSL value. It is believed that drivers who are significantly speeding are relatively aggressive drivers who may not follow VSL. Besides, at the high-speed limit, these drivers will be speeding by more than $30 \%$, while the percentage will rise to $50 \%$ at the low VSL value. We believe that some drivers might challenge the rationality of VSL control when a low-speed limit is implemented, whereby the reduced VSL obedience rate leads to the phenomenon of higher mean speeds under lower VSL values.

2.3. Average Desired Speed. The average desired speed reflects the compliance rate of drivers to some extent. In order to quantify the change in the average expected speed, the arithmetic average speed is calculated in the case of different speed limit values on different lanes.

Table 1 shows the average desired speed with the headway time greater than $5 \mathrm{~s}$ under different VSL values in each lane ("-" indicates that the number of samples is less than 50; no statistics were performed). It can be observed that the driver's desired speed decreased significantly with VSL in all lanes compared with the uncontrolled state. In addition, from the speed limit value of $120 \mathrm{~km} / \mathrm{h}$ to $60 \mathrm{~km} / \mathrm{h}$, the average speed decreases as the variable speed limit decreases. However, the reduction is not linear, since the difference between the driver's desired speed and the VSL value is bigger under the control of the lower VSL value like $60 \mathrm{~km} / \mathrm{h}$.

2.4. Small Headway Distribution. The distribution of the headway, especially the proportion of the smaller headway, is critical to the stability of the traffic flow. This paper mainly focuses on the effect of the variable speed limit on the percentage of vehicles whose headway is less than 1 second. In order to quantify the effects of different VSL values on the headway, we calculated the percentage of small headways within different density intervals under different speed limits on different lanes. Table 2 summarizes the percentage of vehicles with smaller than $1 \mathrm{~s}$ headway under all combinations of density intervals and speed limits on the left, the middle, and the right lane ("-" indicates that less than 300 vehicles were observed within the associated density interval).

It can be observed from the table that the ratio of less than $1 \mathrm{~s}$ headway in the uncontrolled state increases with the increase of the density, while the ratio under the VSL control does not change significantly with the increase of the density. In general, the percentage of less than $1 \mathrm{~s}$ headway in the VSL control is obviously lower than that in the uncontrolled state. The percentage at the speed limit of $100 \mathrm{~km} / \mathrm{h}$ and $80 \mathrm{~km} / \mathrm{h}$ is the least, not even less than $50 \%$ of the uncontrolled state. The results show that some drivers become more cautious under VSL control and tend to maintain a large headway to ensure safety.

\section{Single-Lane Cellular Automaton Model}

This section presents a single-lane cellular automaton model for variable speed limit (VSL) control. On the basis of improved cellular automaton model incorporating impaired driver's radical feature (RF) raised by Qu et al. [29], this paper introduces the VSL compliance parameter to describe the driver's compliance characteristics, setting the desired speed according to the compliance rate of each vehicle, and then modify the moderation probability of the RF model combined with compliance rate; that is, if a driver with VSLs compliance and within the range of the VSLs control keeps headway less than 1 second, the driver will possess higher decelerating probability.

In the model, the driver will possess a higher decelerating probability while being within the range of the VSL control with headway less than 1 second. The model was established under two assumptions:

(1) The VSL control changes the desired speed of most drivers, but the desired speed and the value of VSL are not equal and their relationship is not linear.

(2) The ratio of the smaller headway (less than $1 \mathrm{~s}$ ) is significantly reduced under the VSL control [24], which reveals that some drivers become more cautious in driving and tend to maintain a larger headway to ensure safety.

The random probability of deceleration expression is 


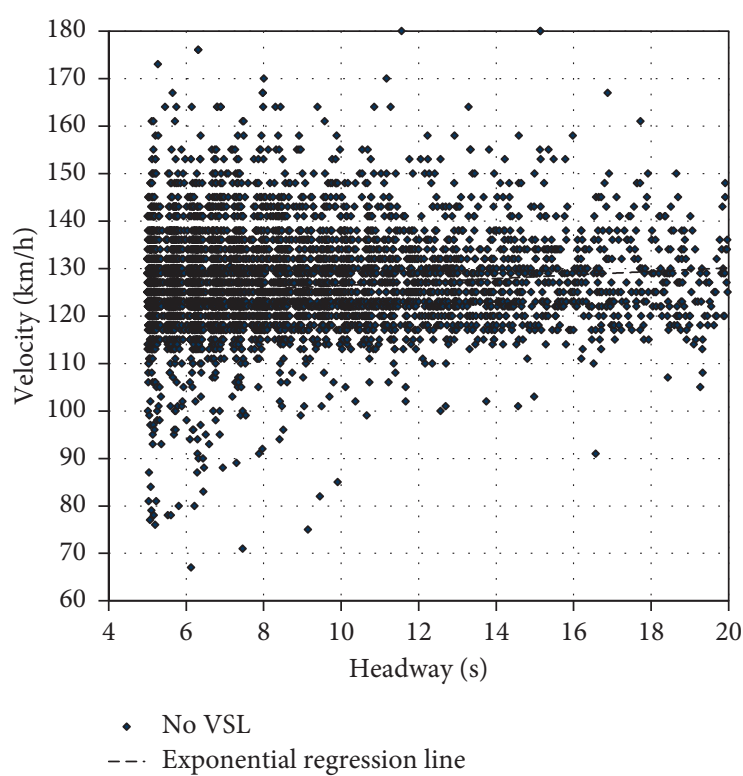

(a)

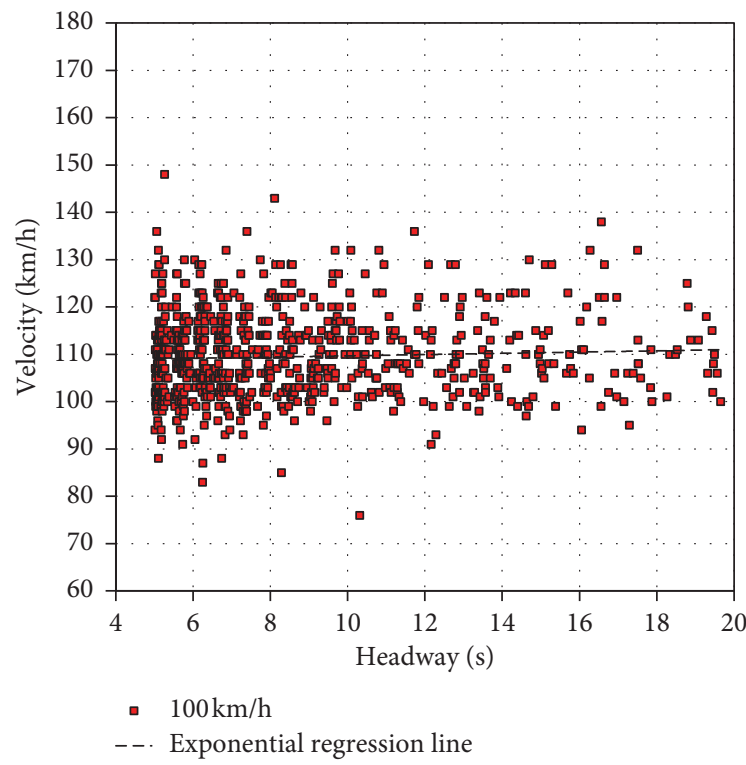

(c)

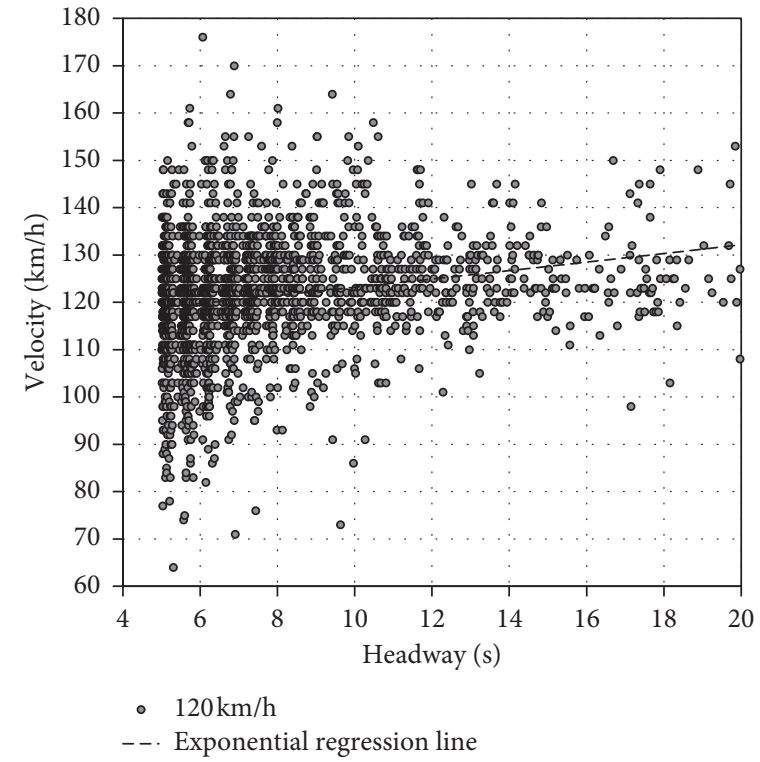

(b)

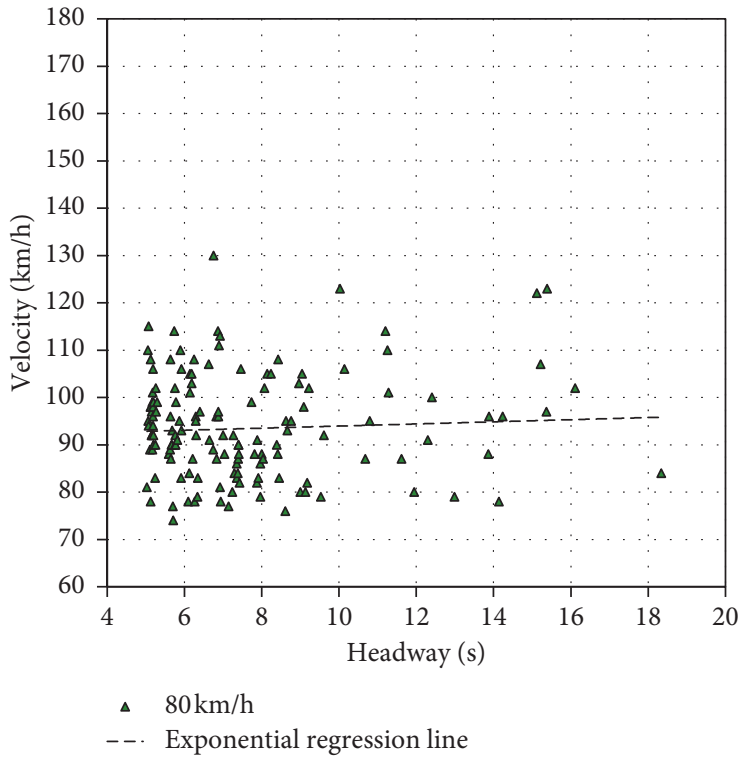

(d)

Figure 1: Speed headway of the vehicles on the left lane (headway greater than $5 \mathrm{~s}$ ): (a) uncontrolled state, (b) $120 \mathrm{~km} / \mathrm{h},(\mathrm{c}) 100 \mathrm{~km} / \mathrm{h}$, and (d) $80 \mathrm{~km} / \mathrm{h}$.

$$
P_{\text {slow }}\left(v_{n}(t), b_{n+1}(t), t_{h}, t_{s}, c_{n}, a_{n}(t)\right)=\left\{\begin{array}{l}
p_{b}: \text { if } b_{n+1}=1 \text { and } t_{h}<t_{s} \\
p_{0}: \text { if } v_{n}=0 \\
p_{c}: \text { if } c_{n}=1 \text { and } a_{n}(t)=1 \text { and } t_{h}<1 \\
p_{d}: \text { in all other cases, }
\end{array}\right.
$$

where $c_{n}$ is the compliance parameter of $n^{\text {th }}$ vehicle, $c_{n}=1$ means compliance, $c_{n}=0$ indicates noncompliance, the compliance of vehicle is affected by the driver's personal characteristics, and the compliance property of each vehicle is initially determined and will not change along with the simulation or varies after each update circle. The compliance parameter of each vehicle is determined by the following formula:

$$
c_{n}=\left\{\begin{array}{l}
1: \text { if } r \text { and }(0,1)<C \\
0: \text { else }
\end{array}\right.
$$


TABLE 1: The average vehicle speed with a headway greater than $5 \mathrm{~s}$ at different VSLs in each lane.

\begin{tabular}{|c|c|c|c|c|c|c|}
\hline \multirow{2}{*}{ Lane } & \multicolumn{6}{|c|}{ Average desired speed $(\mathrm{km} / \mathrm{h})$} \\
\hline & No VSL & $120 \mathrm{~km} / \mathrm{h}$ & $100 \mathrm{~km} / \mathrm{h}$ & $80 \mathrm{~km} / \mathrm{h}$ & $60 \mathrm{~km} / \mathrm{h}$ & $50 \mathrm{~km} / \mathrm{h}$ \\
\hline Left lane & 126.9 & 121.8 & 109.8 & 94.0 & 86.1 & - \\
\hline Middle lane & 114.9 & 105.0 & 99.0 & 85.1 & 80.2 & - \\
\hline Right lane & 97.0 & 89.9 & 88.3 & 78.8 & 74.4 & - \\
\hline
\end{tabular}

TABle 2: Percentages of smaller than $1 \mathrm{~s}$ headway within different density intervals under different speed limits.

\begin{tabular}{|c|c|c|c|c|c|c|c|}
\hline \multirow{2}{*}{ Lane } & \multirow{2}{*}{ Density intervals (veh/km) } & \multicolumn{6}{|c|}{ Percentage of less than $1 \mathrm{~s}$ headway under different speed limits } \\
\hline & & No VSL & $120 \mathrm{~km} / \mathrm{h}$ & $100 \mathrm{~km} / \mathrm{h}$ & $80 \mathrm{~km} / \mathrm{h}$ & $60 \mathrm{~km} / \mathrm{h}$ & $50 \mathrm{~km} / \mathrm{h}$ \\
\hline \multirow{5}{*}{ Left lane } & $0-5$ & $24 \%$ & $21 \%$ & $15 \%$ & - & - & - \\
\hline & $5-10$ & $32 \%$ & $28 \%$ & $19 \%$ & $15 \%$ & - & - \\
\hline & $10-15$ & $35 \%$ & $26 \%$ & $14 \%$ & $10 \%$ & - & - \\
\hline & $15-20$ & $37 \%$ & $26 \%$ & $14 \%$ & $11 \%$ & $15 \%$ & $20 \%$ \\
\hline & $20-25$ & $36 \%$ & $29 \%$ & $14 \%$ & $16 \%$ & $18 \%$ & - \\
\hline \multirow{5}{*}{ Middle lane } & $25-30$ & $9 \%$ & $6 \%$ & - & - & - & - \\
\hline & $5-10$ & $15 \%$ & $11 \%$ & $7 \%$ & - & - & - \\
\hline & $10-15$ & $22 \%$ & $16 \%$ & $12 \%$ & $7 \%$ & - & - \\
\hline & $15-20$ & $26 \%$ & $20 \%$ & $14 \%$ & $12 \%$ & $14 \%$ & $14 \%$ \\
\hline & $20-25$ & $23 \%$ & $22 \%$ & $14 \%$ & - & - & - \\
\hline \multirow{4}{*}{ Right lane } & $25-30$ & $3 \%$ & $3 \%$ & - & - & - & - \\
\hline & $5-10$ & $7 \%$ & $6 \%$ & $4 \%$ & $3 \%$ & - & - \\
\hline & $10-15$ & $13 \%$ & $11 \%$ & $5 \%$ & $4 \%$ & $5 \%$ & - \\
\hline & $15-20$ & $18 \%$ & $14 \%$ & - & - & - & - \\
\hline
\end{tabular}

$C$ is the VSL compliance rate, defined as the percentage of the drivers affected by VSL control, abbreviated as "compliance rate" afterward. " $r$ and $(0,1)$ " indicates a random number between $0 \sim 1 . a_{n}(t)$ is a parameter to evaluate whether the vehicle is within the VSL control range, $a_{n}(t)=1$ indicates that the $n^{\text {th }}$ vehicle is within the VSL control range at time $t$, while $a_{n}(t)=0$ indicates that it is out of the range. Other parameters are the same as the RF model defined.

The update rules of the proposed model are as follows:

(1) Determining the desired speed as

$$
\begin{aligned}
& \text { if }\left(c_{n}=1 \text { and } X v s l_{i} \leq x_{n}(t)<X v s l_{i+1}\right), \\
& \text { then: } v_{\text {des }}(n)=\min \left(\bar{v}+\alpha_{n}, v_{\text {max }, n}\right) \quad \text { else } v_{\text {des }}(n)=v_{\text {max }, n} .
\end{aligned}
$$

(2) Determining the probability of randomization as

$$
\begin{aligned}
& P_{\text {slow }}\left(v_{n}(t), b_{n+1}(t), t_{h}, t_{s}, c_{n}, a_{n}(t)\right), \\
& b_{n}(t+1)=0, \\
& a_{n}(\mathrm{t}+1)=0 .
\end{aligned}
$$

(3) Acceleration is

$$
v_{n}(t+1) \longrightarrow \min \left(v_{n}(t)+1, v_{\mathrm{des}}(n)\right)
$$

(4) Deceleration is

$$
\begin{aligned}
& v_{n}(t+1) \longrightarrow \min \left(v_{n}(t), d_{n}^{(\mathrm{eff})}\right) \\
& \operatorname{if}\left(v_{n}(t+1)<v_{n}(t)\right) \text { then: } b_{n}(t+1)=1
\end{aligned}
$$

(5) Randomization is

$$
\begin{aligned}
& \text { if }\left(\operatorname{rand}<p_{\text {slow }}\right), \\
& \text { then: } v_{n}(t+1) \longrightarrow \max \left(v_{n}(t+1)-1,0\right) .
\end{aligned}
$$

(6) Location update is

$$
x_{n}(t+1) \longrightarrow x_{n}(t)+v_{n}(t+1) \text {. }
$$

(7) Determining whether it is in the range of VSL control as

$$
\begin{aligned}
& \text { if }\left(X v s l_{i} \leq x_{n}(t+1)<X v s l_{i+1} \text { and } V v s l_{i} \neq 0\right) \text {, } \\
& \text { then: } a_{n}(t+1)=1,
\end{aligned}
$$

where $X v s l_{i}$ is the starting position of the $i^{\text {th }}$ VSL control area. $\bar{v}$ denotes the average value of vehicle's desired speed (AVDS) under the VSL control, determined by the speed limit value in the $i^{\text {th }}$ VSL control area, which is called the AVDS of VSL, described as $\bar{v}=f(V v s l i)$. $V v s l_{i}$ indicates the 
speed limit value in the $i^{\text {th }}$ VSL control area. $v_{\text {des }}(n)$ is the desired speed of the $n^{\text {th }}$ vehicle. $v_{\max , n}$ is the maximum speed of the $n^{\text {th }}$ vehicle. The other parameter definitions are the same as the comfortable driving (CD) model [30].

\section{Model Calibration}

4.1. AVDS and Average Maximum Speed (AMS) under VSL. In this study, the left lane was selected as the calibration target lane for the single-lane model. The vehicle's desired speed and the VSL values are neither equal nor linear under the VSL control. Therefore, the AVDS under the different VSL control cases needs to be calibrated according to the actual data. On the basis of a number of samples, the average speed of the vehicle with headway larger than 5 seconds can be selected as the value of AVDS under a certain speed limit control. If it is not possible to obtain a sufficient sample size, the average speed in the lower density state may be selected as the value of AVDS. In this study, the AMS is equivalent to the AVDS under the condition of no VSL control, and the calculation method is the same as in calculating AVDS.

For the speed limit values of $120 \mathrm{~km} / \mathrm{h}, 100 \mathrm{~km} / \mathrm{h}, 80 \mathrm{~km} / \mathrm{h}$, and $60 \mathrm{~km} / \mathrm{h}$, we selected the average speed of the vehicle with headway greater than 5 seconds as the AVDS of the VSL control. For the $50 \mathrm{~km} / \mathrm{h}$ speed limit value, the average speed in the lower density state was selected as its AVDS. The specific values are shown in Table 3.

The result of analyzing exiting data reveals that the formation of regression function is consistent with quadratic polynomial; in order to supplement the missing values to calculate the corresponding AVDS, the quadratic polynomial regression can be performed through the existing data, and the values are listed in Table 3. Herein, only the speed limit values, which can be integers divided by 10 and greater than $50 \mathrm{~km} / \mathrm{h}$, are listed. The regression function is modeled as (10), where $x$ is cell speed value and $y$ is the corresponding AVDS. The coefficient of determination is 0.97 :

$$
y=0.0010 x^{2}-1.213 x+126.3
$$

4.2. Value of Compliance Rate. The value of the compliance rate is affected by many factors, among which the VSLs control traffic regulations and the driver's recognition are the most significant. In general, the compliance rate under the mandatory regulations is superior to recommended regulations and is more prominent along with the penalty rate rising or driver's recognition improvement.

There are some drawbacks of analyzing compliance through a survey. Firstly, it depends on whether the driver answers the questionnaire honestly. What is more, driving behavior may be different between humans. Therefore, in this paper, the statistics method was conducted. Through observing the distribution of vehicle speeds with its headway larger than 5 seconds, we found that some vehicles' speeds are significantly higher than others in any speed limit control. These vehicles have noncompliance characteristics because they were not affected by VSL control cases.
It can be concluded that, among all vehicles with headway larger than 5 seconds, except for some vehicles with speed beyond the critical value, the percentage of the remaining vehicles is the compliance rate of VSL control. The critical speed value should be kept in a reasonable scope to decrease the errors in statistical analysis; in this study, the average speed over $30 \%$ of the limit value was selected. In the case of a $100 \mathrm{~km} / \mathrm{h}$ limit control situation, according to statistics results, $5 \%$ of vehicles were beyond $130 \mathrm{~km} / \mathrm{h}$. As a consequence, the compliance rate is $95 \%$, drafted as $C=95 \%$.

\subsection{Calibration of Random Probability of Deceleration $p_{c}$.} The random probability of deceleration $p_{c}$ is to simulate the driver's cautious driving characteristics under the VSL control; its value will directly affect the proportion of the small headway. Therefore, we will calibrate the parameter $p_{c}$ by determining the ratio of the headway less than 1 second in the actual VSL control.

To calibrate the $p_{c}$ values corresponding to different speed limit values, we performed simulations under speed limit values of $120 \mathrm{~km} / \mathrm{h}, 100 \mathrm{~km} / \mathrm{h}, 80 \mathrm{~km} / \mathrm{h}, 60 \mathrm{~km} / \mathrm{h}$, and $50 \mathrm{~km} / \mathrm{h}$, respectively. For a certain speed limit value, the value of the $p_{c}$ is gradually changed, and other parameters were fixed. The road length was set to 4000 cell units, the time step was set to 1 second, and the density was set to $15 \mathrm{veh} / \mathrm{km}$, respectively. Periodic boundary conditions were adopted. The initial position and speed of the vehicle were randomly generated. Each simulation runs 10,600 time steps, where the first 10,000 time steps were not counted in order to eliminate the effects of transients, while in the next 600 time steps, the ratio of the headway less than 1 second was calculated. For the same $p_{c}$ value, the simulation was taken 10 times to obtain an average value. Figure 2 shows the ratio of the headway less than 1 second at different $p_{c}$ values under the $100 \mathrm{~km} / \mathrm{h}$ speed limit control.

It can be seen from the figure that the ratio of the headway less than 1 second is relatively sensitive to the parameter $p_{c}$. Qu et al. [24] mentioned that, in practice, the ratio of the headway less than 1 second under the control of $100 \mathrm{~km} / \mathrm{h}$ speed limit in the free-flow state is about $15 \%$ 20\%; therefore, the value of $p_{c}$ ranges from approximately 0.5 to 0.8 under the limit speed value of $100 \mathrm{~km} / \mathrm{h}$. In the same way, we calibrate the $p_{c}$ values of other speed limit values. The results are shown in Table 4.

As can be seen from the results, the $p_{c}$ values corresponding to the excessively high- and low-speed limit values are small, while under the moderate speed limit, values $(80 \mathrm{~km} / \mathrm{h}, 100 \mathrm{~km} / \mathrm{h})$ are large. This reveals that the driver's cautious driving behavior under the control of the moderate speed limit is the most obvious. For the sake of simplicity, this study divides the value of the parameter $p_{c}$ into two parts: when the speed limit value is between $80 \mathrm{~km} / \mathrm{h}$ and $100 \mathrm{~km} / \mathrm{h}, p_{c}=0.6$; under other speed limit values, $p_{c}=0.4$.

\section{Numerical Simulation of the Model}

In this section, the simulation of a fundamental diagram, time-space diagram, and speed differences characteristics was conducted to evaluate the performance of the singlelane cellular automaton model. In the simulation, the length of the road was set to 4000 cell units, and the time step was 
TABLE 3: Variable speed limit AVDS values of the left lane.

\begin{tabular}{lcc}
\hline $\begin{array}{l}\text { Speed limit value } \\
(\mathrm{km} / \mathrm{h})\end{array}$ & $\begin{array}{c}\text { AVDS }(\mathrm{km} / \\
\mathrm{h})\end{array}$ & $\begin{array}{c}\text { Corresponding cell speed value } \\
\text { (cell/s) }\end{array}$ \\
\hline No VSL & 126.9 & 23 \\
120 & 121.8 & 22 \\
110 & 113.8 & 21 \\
100 & 109.8 & 20 \\
90 & 98.1 & 18 \\
80 & 94.0 & 17 \\
70 & 90.3 & 17 \\
60 & 86.1 & 16 \\
50 & 90.6 & 17 \\
\hline
\end{tabular}

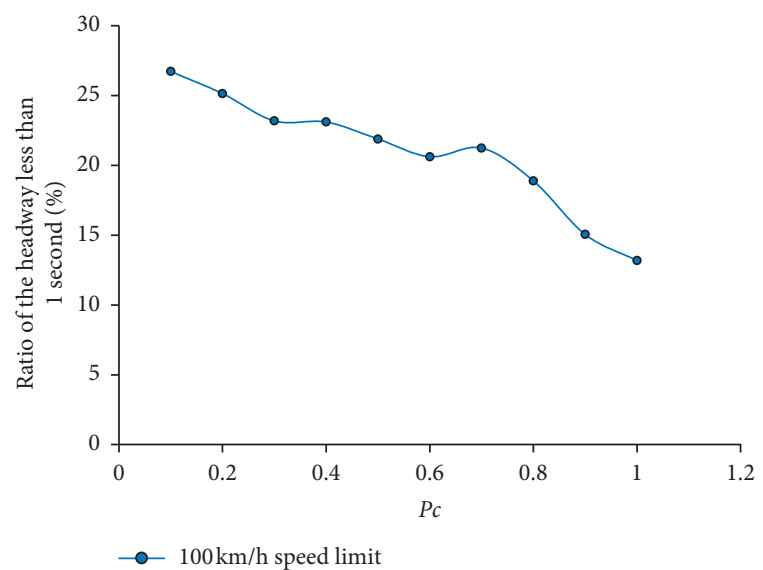

Figure 2: Proportion of the headway less than 1 second at different $p_{c}$ values.

TABle 4: Range of values of different VSL parameters, $p_{c}$.

\begin{tabular}{lc}
\hline Speed limit value $(\mathrm{km} / \mathrm{h})$ & The range of $p_{c}$ \\
\hline 120 & $0.3-0.5$ \\
100 & $0.5-0.9$ \\
80 & $0.5-0.8$ \\
60 & $0.3-0.6$ \\
50 & $0.3-0.5$ \\
\hline
\end{tabular}

set to 1 second with periodic boundary condition. The initial position and speed of the vehicle were randomly generated. Each simulation ran 10,600 time steps, of which the first 10,000 time steps did no statistics to eliminate the impact of the transient. The value for the new model's parameters is the same as that in the calibration session.

5.1. Fundamental Diagram. This study carried out a pseudonumerical simulation of VSL control with values of $120 \mathrm{~km} /$ $\mathrm{h}, 100 \mathrm{~km} / \mathrm{h}$, and $80 \mathrm{~km} / \mathrm{h}$ with no VSL control, which has been run 10 times for each density value; then the average traffic flow was calculated. The fundamental diagram under different speed limit values is shown in Figure 3. It can be seen from the figure that, in the low-density region, the average speed (curve slope) under the VSL control is minor compared to that of the uncontrolled situation, with the tendency of the speed limit value and the average speed being the same. In the high-density region, it can be found

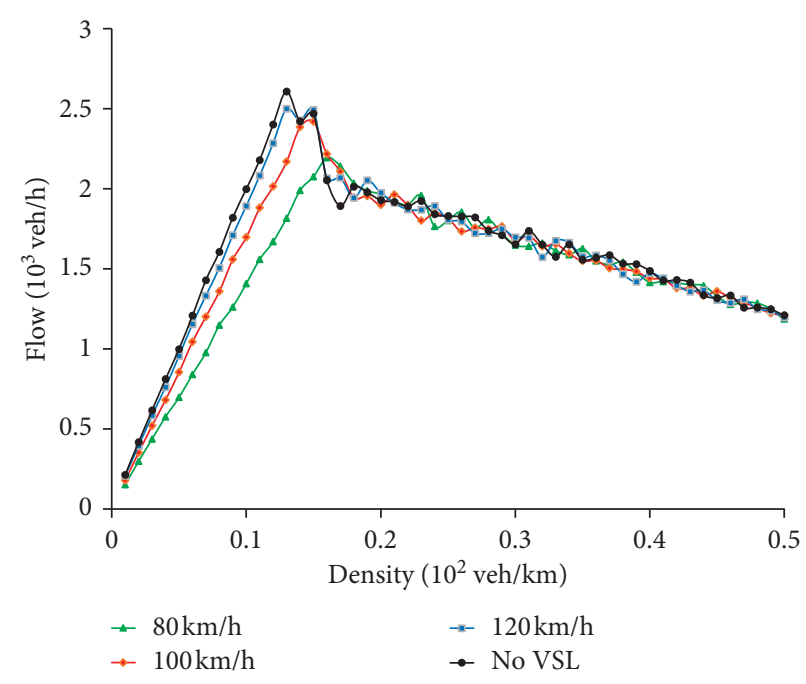

Figure 3: Fundamental diagram of different speed limit values for single-lane cellular automaton model simulation.

that the fundamental diagram at different speed limit values does not significantly differ from the uncontrolled situation. In the moderate density region, the critical density (the density region where the traffic flow changes phase) under the VSL control is larger than that in an uncontrolled situation, and the corresponding key density value is growing with the descent of the speed limit value. Accompanied by the decrease of speed limit value $(80 \mathrm{~km} / \mathrm{h})$, the characteristics of the traffic flow phase transition have become vague. In addition, under the VSL control, the road capacity declined with its scope being weightier as the speed limit value decreased. The features of the fundamental diagram in the above simulations are basically consistent with those in actual observation.

5.2. Time-Space Diagram. Figure 4 shows the time-space diagram of the $100 \mathrm{~km} / \mathrm{h}$ speed limit and no VSL control cases at $0.1,0.15$, and 0.3 density, respectively. It can be seen from the figure that, in the small density $(k=0.1)$ and large density $(k=0.3)$ regions, the VSL control does not significantly change the basic form of the time-space diagram. In the small density region, the traffic flow is in the free-flow state as a form of the vehicle fleet, while the large density region exhibits a wide motion-blocking feature with a largescale blockage group. In the moderate density $(k=0.15)$ region, the VSL control significantly changes the shape of the time-space diagram, because it does not exhibit a phase separation phenomenon consisting of a distinct blocking phase and a moving phase compared to the uncontrolled situation, but possesses free-flow characteristics. It can be confirmed in Figure 4 that when $k=0.15$, the free-flow state changes to the blocking phase without VSL control and maintains stability while being under control. This corresponds with a previous study reporting that VSL can increase the critical density [12].

It can be found that, for a certain speed limit value, the spatiotemporal characteristics changed every time, but some 


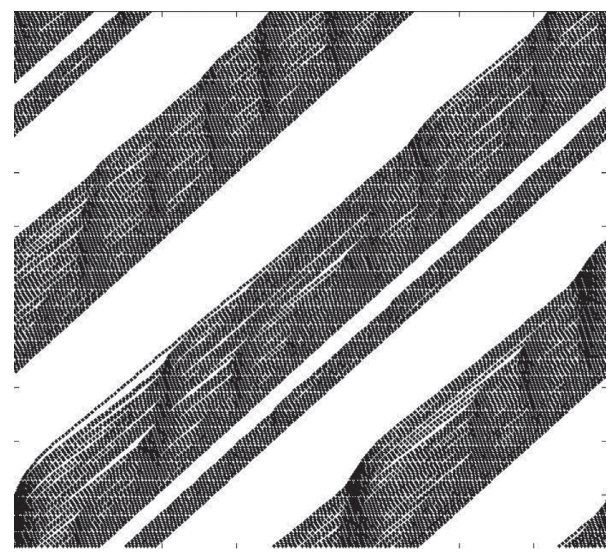

(a)

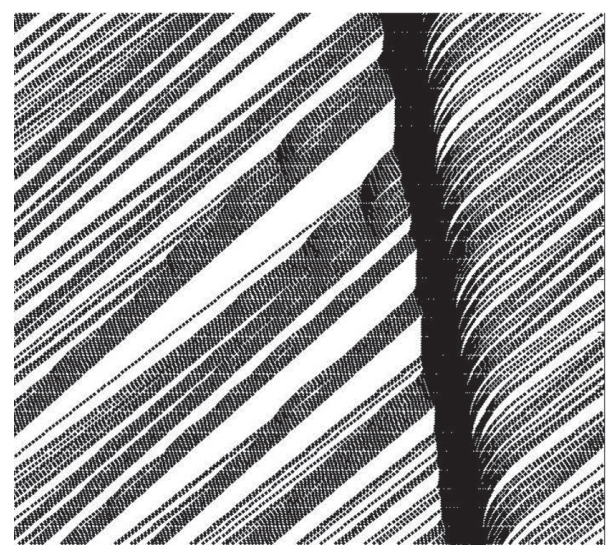

(c)

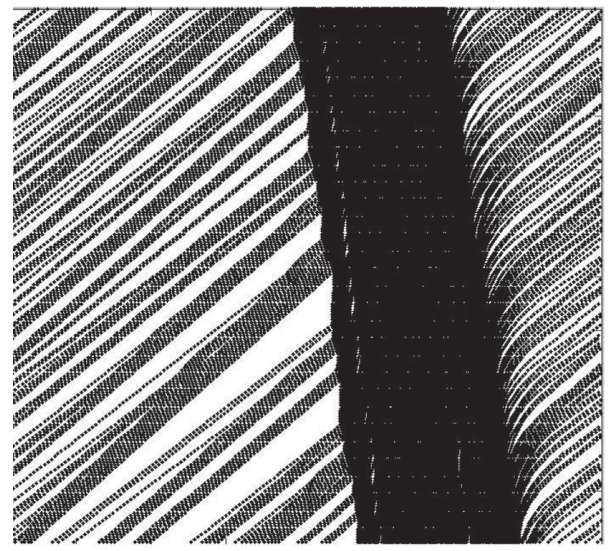

(e)

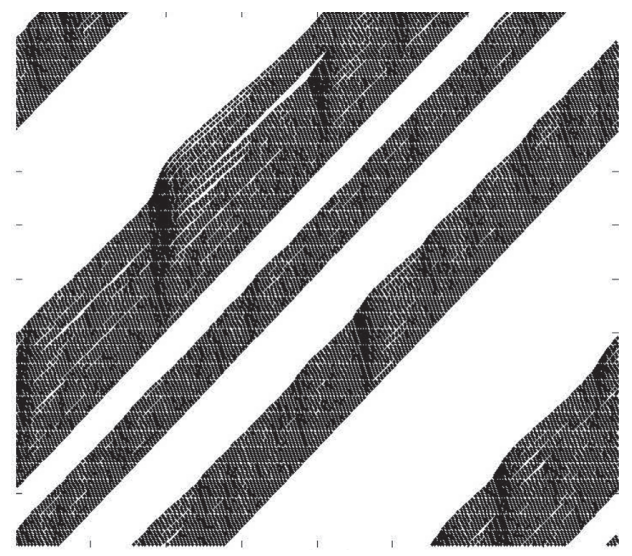

(b)

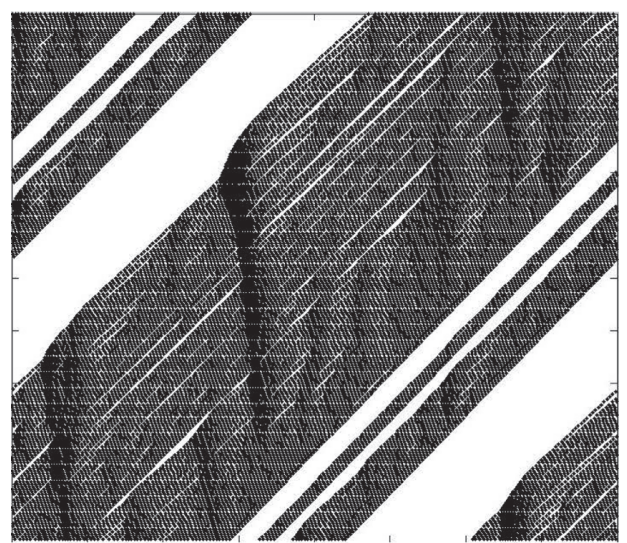

(d)

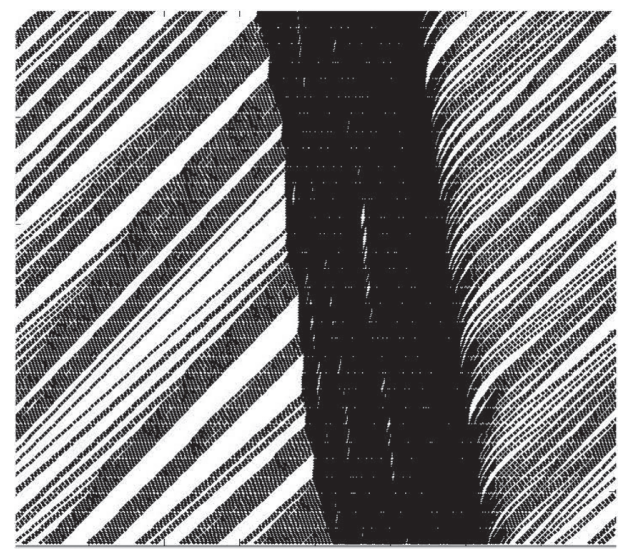

(f)

Figure 4: Time-space diagrams for controlled and uncontrolled situations. (a, c, e) Time-space diagrams at $k=0.1,0.15$, and 0.3 density in uncontrolled state. (b, d, e) Time-space diagrams of $\mathrm{VSL}=100 \mathrm{~km} / \mathrm{h}$ at $k=0.1,0.3$, and 0.5 density.

regulations existed. To explore the discipline, we carried out simulations to calculate the frequency of the free-flow state. On different density values near the critical density, 20 times of simulations under each speed limit value were conducted, the results are shown in Table 5.

The result reveals that the speed limit value decreased as the density increased, and the probability of generating a free-flow state expanded. This indicates that the VSL control could boost the critical density of traffic flow, which is consistent with the fundamental diagram and the actual observation of traffic flow characteristics.

5.3. Average Speed Difference of Two Adjacent Vehicles. The average speed difference (ASD) of two adjacent vehicles was selected to measure the speed difference characteristics of traffic flow. It is defined as the mean value of speed 
TABle 5: Proportion of free-flow characteristics at different density limits in time-space diagrams.

\begin{tabular}{lccccc}
\hline Density value $(\mathrm{veh} / \mathrm{km})$ & No VSL $(\%)$ & $120 \mathrm{~km} / \mathrm{h}(\%)$ & $100 \mathrm{~km} / \mathrm{h}(\%)$ & $80 \mathrm{~km} / \mathrm{h}(\%)$ & $60 \mathrm{~km} / \mathrm{h}(\%)$ \\
\hline 0.14 & 100 & 100 & 100 & 85 & 100 \\
0.15 & 25 & 30 & 35 & 100 & 100 \\
0.16 & 10 & 5 & 10 & 95 & 100 \\
0.17 & 0 & 0 & 0 & 0 & 100 \\
0.18 & 0 & 0 & 0 & 0 & 20 \\
0.19 & 0 & 0 & 0 & 0 & 0 \\
0.20 & 0 & & 5 & 0 & 0 \\
\hline
\end{tabular}

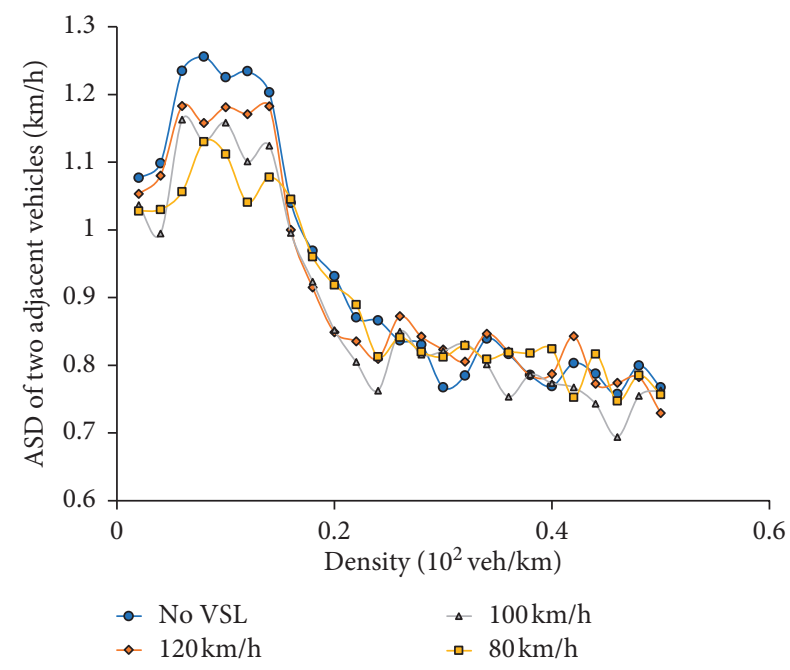

Figure 5: ASD of two adjacent vehicles in different speed limit values and different density conditions.

difference between the adjacent vehicles passing a given point at a specified time interval [31], as shown as follows:

$$
\mathrm{ASD}=\overline{\Delta v}=\frac{\sum_{n=1}^{N-1}\left|v_{n}-v_{n+1}\right|}{n-1}
$$

where $v_{n}$ represents the speed of the $n^{\text {th }}$ vehicle when passing the fixed point and $N$ represents the number of vehicles passing a given point. In this study, simulations were carried out 10 times for each density condition following the singlelane cellular automaton model. Through simulations, the ASD of two adjacent vehicles in different speed limit values and different density conditions is shown in Figure 5.

It can be seen from the figure that the ASD of two adjacent vehicles in different VSL control cases is basically similar to the trend of the density varying. Except for excessive small density conditions, the ASD of two adjacent vehicles decreased with the density growth. In the small density region, the value under the VSL control is less than that in an uncontrolled state; this tendency is similar to actual situations [24]. In the large density region, different speed limit values are not significantly different.

\section{Conclusions}

This paper studies the real driving behavior data under VSL control and finds the following conclusions:
(1) Through the processing and analysis of traffic big data, the traffic data in the same density interval with different VSL control was compared with that without VSL control to investigate the effect of VSL control on driving behavior

(2) Under the control of the VSL, there always exists a certain proportion of drivers who fail to comply with the VSL control

(3) The VSL control changes the driver's desired speed, but the average desire speed and the value of VSL are not equal and show a nonlinear relationship

(4) The proportion of smaller headway time (less than $1 \mathrm{~s})$ under VSL control is significantly reduced, which shows that some drivers become more cautious under the VSL control

Based on the above-mentioned driving behavior, this study introduces the compliance rate parameter during the modeling process. In addition, it improves the slowing probability of the RF model [26], so that the vehicle slows down with a higher probability when the headway time is less than $1 \mathrm{~s}$, in order to simulate the prudent driving behavior of the vehicle under the VSL control. In this study, the parameters of the model are calibrated using real vehicle data under VSL control. The numerical simulation results show that the model can not only fit the macroscopic traffic phenomena such as the fundamental diagrams and the time-space diagrams under the VSL control, but also fit the influence of the VSL on the speed dispersion.

It should be noted that this study assumed that the driver's radical feature is subject to the normal distribution when defining the function of radical feature parameter. Whether this assumption is reasonable or not needs to be discussed further in future research. Besides, this study is just early stage work to incorporate impaired driving behavior. More work should be done to develop a more accurate calibration process as well as multiple lane models. In our future research, we will focus on collecting real data to help discuss the driver's radical degree and its distribution under the car-following state.

\section{Data Availability}

The basic data used to support the findings of this study are available from the corresponding author upon request. 


\section{Conflicts of Interest}

The authors declare that there are no conflicts of interest regarding the publication of this paper.

\section{Acknowledgments}

This research was supported by the National Key R\&D Program in China (Grant no. 2018YFB1600600), the MOE (Ministry of Education in China) Project of Humanities and Social Sciences (Project no. 20YJAZH083), and the National Natural Science Foundation of China (Grant no. 51878161).

\section{References}

[1] C. Levecq, B. Kuhn, and D. Jasek, "General guidelines for active traffic management deployment," Interim Report, Texas A\&M Transportation Institute, College Station, TX, USA, 2011.

[2] Y. Zhang and P. A. Ioannou, "Combined variable speed limit and lane change control for highway traffic," IEEE Transactions on Intelligent Transportation Systems, vol. 18, no. 7, pp. 1812-1823, 2017.

[3] R. C. Carlson, I. Papamichail, and M. Papageorgiou, "Local feedback-based mainstream traffic flow control on motorways using variable speed limits," IEEE Transactions on Intelligent Transportation Systems, vol. 12, no. 4, pp. 1261-1276, 2011.

[4] M. Yu and W. "David" Fan, "optimal variable speed limit control in connected autonomous vehicle environment for relieving freeway congestion," Journal of Transportation Engineering, Part A: Systems, vol. 145, no. 4, Article ID 04019007, 2019.

[5] H.-Y. Jin and W.-L. Jin, "Control of a lane-drop bottleneck through variable speed limits," Transportation Research Part C: Emerging Technologies, vol. 58, pp. 568-584, 2015.

[6] M. Hajiahmadi, G. S. van de Weg, C. M. J. Tampere et al., "Integrated predictive control of freeway networks using the extended link transmission model," IEEE Transactions on Intelligent Transportation Systems, vol. 17, no. 1, pp. 65-78, 2016.

[7] A. Csikós and B. Kulcsár, "Variable speed limit design based on mode dependent Cell Transmission Model," Transportation Research Part C: Emerging Technologies, vol. 85, pp. 429-450, 2017.

[8] M. Kontorinaki, I. Karafyllis, and M. Papageorgiou, "Global exponential stabilisation of acyclic traffic networks," International Journal of Control, vol. 92, no. 3, pp. 564-584, 2019.

[9] J. R. José, I. Papamichail, M. Papageorgiou, and B. De Schutter, "Macroscopic modeling of variable speed limits on freeways," Transportation Research Part C: Emerging Technologies, vol. 100, pp. 15-33, 2019.

[10] S. Smulders, "Control by variable speed signs: the Dutch experiment," in Proceedings of the 6th International Conference on Road Traffic Monitoring and Control, London, UK, 1992.

[11] M. Cremer, "Der verkehrsfluss auf schnellstraßen," in Modelle, Überwachung, Regelung, Springer-Verlag, Berlin, Germany, vol. 3.Theoretical Computer Science, 1979.

[12] M. Papageorgiou, E. Kosmatopoulos, and I. Papamichail, "Effects of variable speed limits on motorway traffic flow," Transportation Research Record: Journal of the Transportation Research Board, vol. 2047, no. 1, pp. 37-48, 2008.
[13] A. Hegyi, B. De Schutter, and H. Hellendoorn, "Model predictive control for optimal coordination of ramp metering and variable speed control *," in Proceedings of the 1st European Symposium on Intelligent Technologies, Hybrid Systems and their implementation on Smart Adaptive Systems (EUNITE 2001), pp. 1-6, Tenerife, Spain, December 2001.

[14] I. Papamichail, K. Kampitaki, M. Papageorgiou, and A. Messmer, "Integrated ramp metering and variable speed limit control of motorway traffic flow," IFAC Proceedings Volumes, vol. 41, no. 2, pp. 14084-14089, 2008.

[15] C. Lee, B. Hellinga, and F. Saccomanno, "Evaluation of variable speed limits to improve traffic safety," Transportation Research Part C: Emerging Technologies, vol. 14, no. 3, pp. 213-228, 2006.

[16] A. Torday and M. Bierlaire, "Papabiles: simulation-based evaluation of the impact of telematics in the lausanne area: a pilot study," in Proceedings of the Swiss Transport Research Conference, Ascona, Switzerland, March 2001.

[17] S. Yadlapati and B. Park, "Development and testing of variable speed limit control logics for work zones using simulation," Center for Transportation Studies at the University of Virginia, Charlottesville, VA, USA, 2004.

[18] V. Milanés and S. E. Shladover, "Handling cut-in vehicles in strings of cooperative adaptive cruise control vehicles," Journal of Intelligent Transportation Systems, vol. 20, no. 2, pp. 178-191, 2016.

[19] V. Milanes, S. E. Shladover, J. Spring, C. Nowakowski, H. Kawazoe, and M. Nakamura, "Cooperative adaptive cruise control in real traffic situations," IEEE Transactions on Intelligent Transportation Systems, vol. 15, no. 1, pp. 296-305, 2014.

[20] V. Milanés and S. E. Shladover, "Modeling cooperative and autonomous adaptive cruise control dynamic responses using experimental data," Transportation Research Part C: Emerging Technologies, vol. 48, pp. 285-300, 2014.

[21] X. Y. Lu and S. Shladover, "MPC-based variable speed limit and its impact on traffic with V2I type ACC," in 2018 21st International Conference on Intelligent Transportation Systems (ITSC), pp. 3923-3928, Maui, HI, USA, 2018 November.

[22] M. Morari and J. H. Lee, "Model preditive control: past, present and future," Neuroscience, vol. 23, pp. 667-682, 2014.

[23] J. Ma, X. Li, S. Shladover et al., "Freeway speed harmonization," IEEE Transactions on Intelligent Vehicles, vol. 1, no. 1, pp. 78-89, 2016.

[24] X. Qu, W. Wang, B. Ran, and Y. Dai, "Quantitative analysis of the impact of variable speed limits on motorway safety," in Proceedings of the Transportation Research Board 96th Annual Meeting, Washington DC, USA, 2017.

[25] S. Li, P. Li, Y. Yao, X. Han, Y. Xu, and L. Chen, "Analysis of drivers' deceleration behavior based on naturalistic driving data," Traffic Injury Prevention, vol. 21, no. 1, pp. 42-47, 2020.

[26] R. Hao, H. Yang, and Z. Zhou, "Driving behavior evaluation model base on big data from internet of vehicles," International Journal of Ambient Computing and Intelligence, vol. 10, no. 4, pp. 78-95, 2019.

[27] Z. Deng, D. Chu, C. Wu, Y. He, and J. Cui, "Curve safe speed model considering driving style based on driver behaviour questionnaire," Transportation Research Part F: Traffic Psychology and Behaviour, vol. 65, pp. 536-547, 2019.

[28] N. H. Gartner, C. J. Messer, and A. Rathi, "Traffic flow theorya state-of-the-art report: revised monograph on traffic flow theory," Technical Report, US. Department of transportation, Washington, DC, USA, 2002. 
[29] X. Qu, M. Yang, F. Yang, B. Ran, and L. Li, "An improved single-lane cellular automaton model considering driver's radical feature," Journal of Advanced Transportation, vol. 2018, Article ID 3791820, 10 pages, 2018.

[30] W. Knospe, L. Santen, A. Schadschneider, and M. Schreckenberg, "Towards a realistic microscopic description of highway traffic," Journal of Physics A: Mathematical and General, vol. 33, no. 48, 2000.

[31] H. Wang, "Experimental features and characteristics of speed dispersion in urban freeway traffic," Transportation Research Record: Journal of the Transportation Research Board, vol. 1999, no. 1, pp. 150-160, 2007. 